\title{
Endovascular Treatment of Chronic Mesenteric Ischemia
}

\author{
Yoshimitsu Soga, MD; Hiroyoshi Yokoi, MD; Masashi Iwabuchi, MD; Masakiyo Nobuyoshi, MD
}

\begin{abstract}
Endovascular procedure is frequently used as an alternative to surgical bypass in aortic atherosclerotic lesions. Two rare cases of chronic mesenteric ischemia treated with a stent implantation are reported. The patients were a 72-year-old woman and a 57-year-old woman. They presented with severe abdominal pain after meals, repeatedly. Angiography revealed severe stenosis and occlusion of the super mesenteric artery (SMA). Endovascular treatment was initially and technically successful in both patients. A Palmaz ${ }^{\circledR}$ stent was deployed in the ostium lesion of the SMA for both cases. Completion angiography showed successful recanalization of the SMA without any complication and with satisfactory distal flow. The patients' symptoms were alleviated. They remained asymptomatic after stent implantation. Primary stenting may be considered a viable alternative to conventional surgery in carefully selected patients with chronic mesenteric ischemia due to SMA stenosis. (Circ J 2008; 72: 1198-1200)
\end{abstract}

Key Words: Chronic mesenteric ischemia; Stent; Superior mesenteric artery stenosis

C hronic mesenteric ischemia (CMI) is a rare disease defined as intestinal ischemia caused by stenosis or occlusion of one or more of the following 3 intestinal arteries: the celiac artery (CA); the superior mesenteric artery (SMA); and the inferior mesenteric artery. The symptoms often manifest as postprandial abdominal pain, nausea, vomiting and epigastralgia. Diagnosis of CMI is difficult and is often delayed. Nonetheless, asymptomatic stenosis of the CA or SMA has been reported in $18 \%$ of patients aged 65 years or older, indicating a high latent prevalence of CMI that suggests the incidence of the disease may increase in the future. Currently, arteriosclerotic CMI is treated using methods such as laparotomy and endovascular therapy, but no specific treatment has been established. Here, we report 2 cases of CMI with stenosis of the SMA diagnosed before treatment, in which good outcomes were obtained using stent placement.

\section{Case Reports}

\section{Case 1}

The patient was a 72-year-old woman, a smoker, and was undergoing oral drug treatment for hypertension and arteriosclerosis obliterans. She was admitted to a clinic for severe postprandial abdominal pain, which had persisted for 6 months and had worsened 1 month before admission. CMI was diagnosed and oral drug treatment was given, but the symptoms did not improve and the patient became unable to ingest food. Severe calcification of the abdominal aorta and the root of the SMA were noted on the abdominal computed tomography (CT) scan, suggesting severe stenosis of the entrance to the SMA. Surgery was not recommended in this instance because of the severe calcification of the aorta, and the patient was transferred to our hospital for endovas-

(Received August 8, 2007; revised manuscript received September 29, 2007; accepted December 5, 2007)

Department of Cardiology, Kokura Memorial Hospital, Kitakyushu, Japan

Mailing address: Masakiyo Nobuyoshi, MD, Department of Cardiology, Kokura Memorial Hospital, 1-1 Kifune-machi, Kokurakita-ku, Kitakyushu 802-8555, Japan. E-mail: kmhptca@nn.iij4u.or.jp All rights are reserved to the Japanese Circulation Society. For permissions, please e-mail: cj@j-circ.or.jp cular treatment. Abdominal angiography detected $99 \%$ stenosis of the root of the SMA (Fig 1A), and CMI associated with severe SMA stenosis was diagnosed.

A 7-Fr sheath was inserted via the left brachial artery, and the SMA was selected using a 5-Fr multipurpose catheter (Terumo, Tokyo, Japan). After passing a 0.014-inch guide wire (Neo's Cruise ${ }^{\mathrm{TM}}$; Getz Bros Co Ltd, Chicago, IL, USA) through the lesion, the lesion was dilated using a balloon catheter (Ultra-Soft ${ }^{\mathrm{TM}} \mathrm{SV} 4.0 \times 20 \mathrm{~mm}$; Boston Scientific, Natick, MA, USA), followed by placement of a stent $\left(\right.$ Palmaz $^{\circledR} 6.0 \times 14 \mathrm{~mm}$; Cordis Johnson \& Johnson, New Brunswick, NJ, USA). Since the root was hard and dilation was insufficient, post-stenting dilation was also performed (POWERFLEX ${ }^{\circledR}$ P3 $6.0 \times 20 \mathrm{~mm}$; Cordis Johnson \& Johnson) at $12 \mathrm{~atm}$ (Fig 1B). The treatment was completed without complications and the patient was discharged after 4 days. The operation took $58 \mathrm{~min}$, the duration of fluoroscopy was $22 \mathrm{~min}$, and the required volume of contrast medium was $160 \mathrm{ml}$.

\section{Case 2}

The patient was a 57-year-old woman who had been undergoing maintenance dialysis treatment for 21 years. She had suffered postprandial abdominal pain for 1.5 years before visiting our hospital, and she had undergone abdominal CT at a clinic. CMI was diagnosed and the patient received oral drug treatment to control intestinal function and for dietary therapy, but the symptoms did not improve and she revisited our hospital. Abdominal CT detected severe calcification of the abdominal aorta and the root of the SMA, and the abdominal aorta was occluded at a site directly above the bifurcation of the common iliac artery. Abdominal angiography detected $100 \%$ occlusion of the root of the SMA, confirming the diagnosis of CMI. Since the patient rejected surgery, catheterization was selected.

After inserting a 6-Fr sheath via the right brachial artery (the left hand was used as the shunt site), the SMA was selectively imaged using a 4-Fr AR1 catheter (Terumo) (Fig 2A). A 0.014-inch guide wire (Runthrough ${ }^{\mathrm{TM}} \mathrm{Ph}$; Terumo) was passed through the lesion, which was then dilated using a balloon catheter with a small diameter (Ryujin ${ }^{\mathrm{TM}}$ Plus $1.25 \times$ $10 \mathrm{~mm}$; Terumo), followed by dilation using a balloon catheter with a larger diameter (Ultra-Soft ${ }^{\mathrm{TM}}$ SV $4.0 \times 10 \mathrm{~mm}$; 

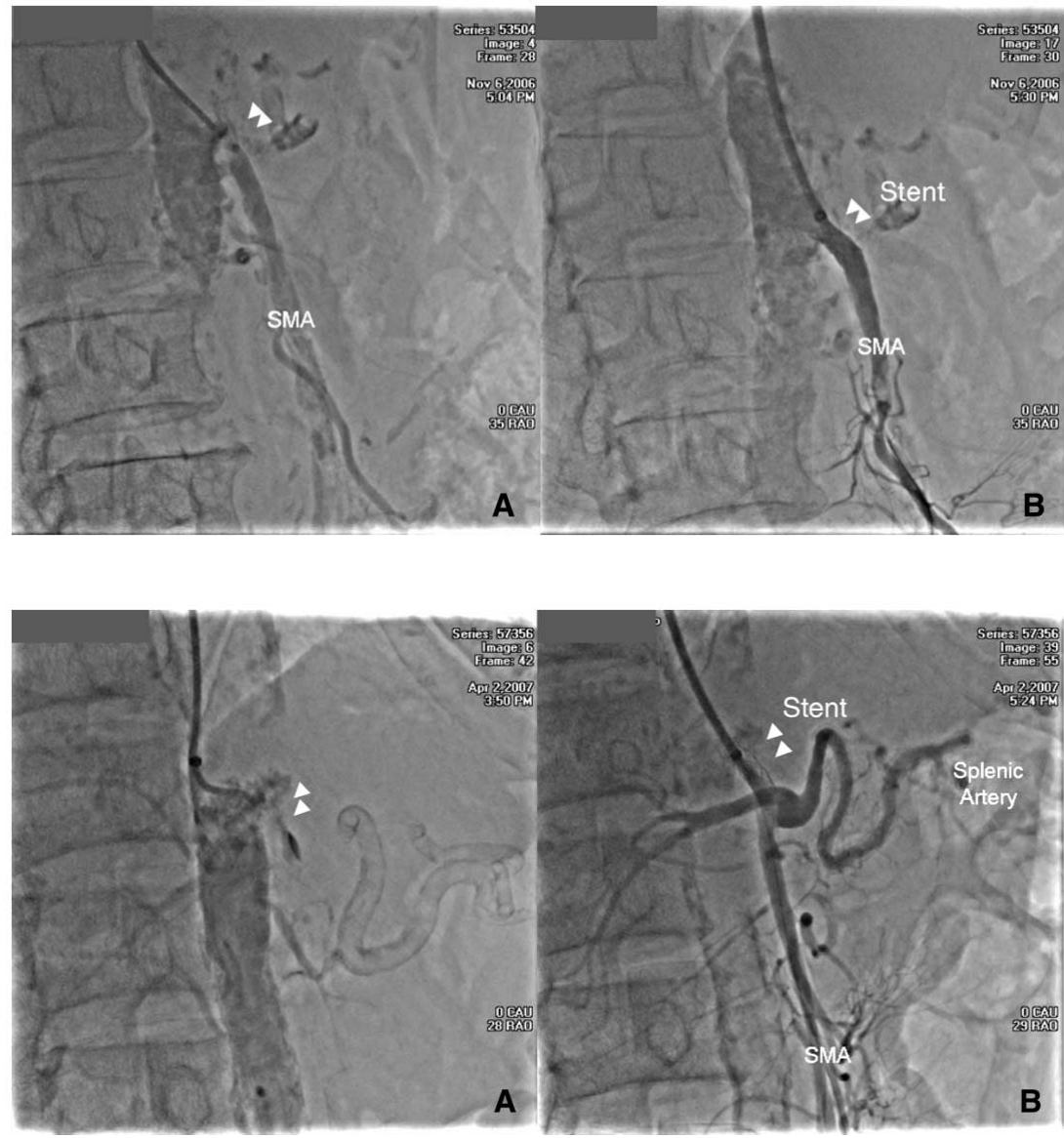

Fig 1. (A) Selective superior mesenteric arteriography showing stenosis in the roof of the superior mesenteric artery (SMA) (arrowheads). (B) Selective superior mesenteric arteriography after treatment with stenting (arrowheads).
Boston Scientific). A stent (Palmaz ${ }^{\circledR}$ Genesis $5.0 \times 18 \mathrm{~mm}$; Cordis Johnson \& Johnson) was placed at $8 \mathrm{~atm}$, post-stenting dilation (AMIIA ${ }^{\mathrm{TM}} 5.0 \times 15 \mathrm{~mm}$; Cordis Johnson \& Johnson) was performed at $12 \mathrm{~atm}$, and the procedure was completed without complication. The SMA shared the root with the CA and the perfusion area was very wide (Fig 2B). The operation took $125 \mathrm{~min}$, the duration of fluoroscopy was $49 \mathrm{~min}$, and the required volume of contrast medium was $360 \mathrm{ml}$. The patient was hospitalized for 6 days.

Each patient received oral aspirin $(200 \mathrm{mg})$ and ticlopidine $(200 \mathrm{mg})$ as antiplatelet agents from more than 2 days before stent placement, and the procedure was initiated after arterial injection of 5,000 U of heparin. The patients started food ingestion on the day after stenting. Abdominal symptoms were dramatically improved, and there has been no recurrence in either patient at 6 months follow up after treatment.

\section{Discussion}

Vomiting, diarrhea, and postprandial abdominal pain occur in many cases of CMI because of stenosis or occlusion of 2 or more visceral blood vessels. Generally, CMI is diagnosed by exclusion, which often results in a delayed diagnosis ${ }^{2-4}$ Balloon angioplasty for CMI has been reported occasionally after it was first used in 19805 , but patency in the chronic phase (6-24 months) is generally insufficient (about 50-80\%) ${ }^{6-9}$ The outcome of this procedure has been improved by the development of stents, and a review of recent reports ${ }^{10-15}$ indicates a procedural success rate of $88-100 \%$ and a clinical success rate of $82-100 \%$, with a complication rate of $3-16 \%$. Twelve patients experienced complications in these reports: in $8(67 \%)$ the complication occurred at a vascular access site (false aneurysm and hematoma in 4 and 4 patients, respectively), and arterial dissection, multiorgan failure, contrast medium-associated renal disorder, and arterial occlusion each occurred in 1 case. The clinical success rate in the early phase and the incidence of delayed mesenteric ischemia do not differ significantly between surgery and percutaneous catheterization, 16 but the latter procedure is normally chosen as the first option because of the lower incidence of complications and because it is less invasive. ${ }^{16}$ The clinical success rate is high also in cases of complete occlusion, demonstrating the usefulness of endovascular treatment ${ }^{17}$

In endovascular therapy for SMA occlusion, coaxial insertion of the guide catheter by approaches via the leg is difficult because the SMA sharply branches downward from the aorta and sufficient back-up cannot be obtained. Severe calcification may further increase the difficulty of this procedure, and choice of the stent placement site is difficult also when the lesion is located at the SMA entrance. Therefore, approaches via the arm, and particularly via the left hand, as this requires less bending, may be more appropriate for stent treatment of SMA-associated CMI. This approach may also reduce complications at the punctured site. Improvements in the flexibility and tracking ability of stents and reduction of the stent profile may also promote active application of stent placement and improve outcomes. For example, the effectiveness of primary stenting has been reported in cases with calcification in which balloon angioplasty caused arterial dissection or provided insufficient 
dilation, $3,4,17$

No consensus has been reached regarding stent treatment for CMI but we suggest that this procedure may be the first choice for CMI because of its high success rate and low incidence of complications. However, a high recurrence rate of symptoms has been reported, ${ }^{18}$ and an investigation of the long-term patency of stents is required. In the 2 cases of CMI reported here, we obtained successful outcomes with stenting. These cases are typical, in that diagnosis of CMI was difficult and was delayed by 6 months to 1 year after the onset of subjective symptoms. However, resolution of ischemia may improve symptoms markedly, suggesting that CMI is an appropriate indication for stent treatment.

\section{References}

1. Yamakado K, Takeda K, Nomura Y, Kato N, Hirano T, Matsumura $\mathrm{K}$, et al. Relief of mesenteric ischemia by Z-stent placement into the superior mesenteric artery compressed by the false lumen of an aortic dissection. Cardiovasc Intervent Radiol 1998; 21: 66-68.

2. Matsumoto AH, Angle JF, Spinosa DJ, Hagspiel KD, Cage DL, Leung DA, et al. Percutaneous transluminal angioplasty and stenting in the treatment of chronic mesenteric ischemia: Results and longterm follow-up. J Am Coll Surg 2002; 194: 22-31.

3. Cognet F, Ben Salem D, Dranssart M, Cercueil JP, Weiller M, Tatou $\mathrm{E}$, et al. Chronic mesenteric ischemia: Imaging and percutaneous treatment. Radiographics 2002; 22: 863-879.

4. Sheeran SR, Murphy TP, Khwaja A, Sussman SK, Hallisey MJ. Stent placement for treatment of mesenteric artery stenoses or occlusions. J Vasc Interv Radiol 1999; 10: 861-867.

5. Uflacker R, Goldany MA, Constant S. Resolution of mesenteric angina with percutaneous transluminal angioplasty of a superior mesenteric artery stenosis using a balloon catheter. Gastrointest Radiol 1980; 5: $367-369$.

6. Maspes F, Mazzetti di Pietralata G, Gandini R, Innocenzi L, Lupattelli $\mathrm{L}$, Barzi F, et al. Percutaneous transluminal angioplasty in the treatment of chronic mesenteric ischemia: Results and 3 years of follow-up in 23 patients. Abdom Imaging 1998; 23: 358-363.
7. Golden DA, Ring EJ, McLean GK, Freiman DB. Percutaneous transluminal angioplasty in the treatment of abdominal angina. Am J Roentgenol 1982; 139: 247-249.

8. Matsumoto AH, Tegtmeyer CJ, Fitzcharles EK, Selby JB Jr, Tribble $\mathrm{CG}$, Angle JF, et al. Percutaneous transluminal angioplasty of visceral arterial stenoses: Results and long-term clinical follow-up. J Vasc Interv Radiol 1995; 6: 165-174.

9. Odurny A, Sniderman KW, Colapinto RF. Intestinal angina: Percutaneous transluminal angioplasty of the celiac and superior mesenteric arteries. Radiology 1988; 167: 59-62.

10. Pietura R, Szymanska A, El Furah M, Drelich-Zbroja A, SzczerboTrojanowska M. Chronic mesenteric ischemia: Diagnosis and treatment with balloon angioplasty and stenting. Med Sci Monit 2002; 8: $8-12$.

11. Steinmetz E, Tatou E, Favier-Blavoux C, Bouchot O, Cognet F, Cercueil JP, et al. Endovascular treatment as first choice in chronic intestinal ischemia. Ann Vasc Surg 2002; 16: 693-699.

12. Sharafuddin MJ, Olson CH, Sun S, Kresowik TF, Corson JD. Endovascular treatment of celiac and mesenteric arteries stenoses: Applications and results. J Vasc Surg 2003; 38: 692-698.

13. Chahid T, Alfidja AT, Biard M, Ravel A, Garcier JM, Boyer L. Endovascular treatment of chronic mesenteric ischemia: Results in 14 patients. Cardiovasc Intervent Radiol 2004; 27: 637-642.

14. Resch T, Lindh M, Dias N, Sonesson B, Uher P, Malina M, et al. Endovascular recanalisation in occlusive mesenteric ischemia-feasibility and early results. Eur J Vasc Endovasc Surg 2005; 29: 199 203.

15. Silva JA, White CJ, Collins TJ, Jenkins JS, Andry ME, Reilly JP, et al. Endovascular therapy for chronic mesenteric ischemia. J Am Coll Cardiol 2006; 47: 944-950.

16. Nyman U, Ivancev K, Lindh M, Uher P. Endovascular treatment of chronic mesenteric ischemia: Report of five cases. Cardiovasc Intervent Radiol 1998; 21: 305-313.

17. Landis MS, Rajan DK, Simons ME, Hayeems EB, Kachura JR, Sniderman KW. Percutaneous management of chronic mesenteric ischemia: Outcomes after intervention. J Vasc Interv Radiol 2005; 16: $1319-1325$.

18. Kasirajan K, O'Hara PJ, Gray BH, Hertzer NR, Clair DG, Greenberg $\mathrm{RK}$, et al. Chronic mesenteric ischemia: Open surgery versus percutaneous angioplasty and stenting. J Vasc Surg 2001; 33: 63-71. 